\title{
Cyclotomic FFTs with Reduced Additive Complexities Based on a Novel Common Subexpression Elimination Algorithm
}

\author{
Ning Chen, Student Member, IEEE and Zhiyuan Yan, Senior Member, IEEE
}

\begin{abstract}
In this paper, we first propose a novel common subexpression elimination (CSE) algorithm for matrix-vector multiplications over characteristic-2 fields. As opposed to previously proposed CSE algorithms, which usually focus on complexity savings due to recurrences of subexpressions, our CSE algorithm achieves two types of complexity reductions, differential savings and recurrence savings, by taking advantage of the cancelation property of characteristic-2 fields. Using our CSE algorithm, we reduce the additive complexities of cyclotomic fast Fourier transforms (CFFTs). Using a weighted sum of the numbers of multiplications and additions as a metric, our CFFTs achieve smaller total complexities than previously proposed CFFTs and other FFTs, requiring both fewer multiplications and fewer additions in many cases.
\end{abstract}

Index Terms-Common subexpression elimination (CSE), Complexity theory, Convolution, Discrete Fourier transforms (DFTs), Galois fields, Multiple constant multiplication (MCM), ReedSolomon codes.

\section{INTRODUCTION}

Discrete Fourier transforms (DFTs) over finite fields have widespread applications in error correction coding [1]. For Reed-Solomon codes, all syndrome-based bounded distance decoding methods involve DFTs over finite fields [1]: syndrome computation and the Chien search are both evaluations of polynomials and hence can be viewed as DFTs; inverse DFTs are used to recover transmitted codewords in transformdomain decoders. Thus efficient DFT algorithms can be used to reduce the complexity of Reed-Solomon decoders. For example, using the prime-factor fast Fourier transform (FFT) in [2], Truong et al. proposed [3] an inverse-free transformdomain Reed-Solomon decoder with substantially lower complexity than time-domain decoders; FFT techniques are used to compute syndromes for time-domain decoders in [4].

Using an approach similar to those in previous works (see, for example, [5]), cyclotomic FFT (CFFT) was recently proposed [6] and two variants were subsequently considered [7], [8]. To avoid confusion, we refer to the CFFT proposed in [6] as direct CFFT (DCFFT) and those in [7] and [8] as inverse CFFT (ICFFT) and symmetric CFFT (SCFFT) respectively

This work was supported in part by Thales Communications Inc. and in part by a grant from the Commonwealth of Pennsylvania, Department of Community and Economic Development, through the Pennsylvania Infrastructure Technology Alliance (PITA). The material in this paper was presented in part at the IEEE Workshop on Signal Processing Systems, Shanghai, China, October 2007.

The authors are with the Department of Electrical and Computer Engineering, Lehigh University, Bethlehem, PA 18015, USA (email: nic6@lehigh.edu; yan@lehigh.edu). henceforth in this paper. DCFFT has been shown to be efficient for full DFTs of lengths up to 511 [6], and ICFFT and SCFFT are particularly suitable for partial DFTs, which compute only part of the spectral components and are important for such operations as syndrome computation of Reed-Solomon decoders [7], [8].

Although CFFTs in [6]-[8] achieve low multiplicative complexities, their additive complexities (numbers of additions required) are very high if implemented directly. The methods used in [6]-[8] somewhat alleviate the problem, but the additive complexities of CFFTs in [6]-[8] remain quite high. In this paper, we first propose a novel common subexpression elimination (CSE) algorithm, and then use it to reduce the additive complexities of various CFFTs. The contributions of this paper are:

- To minimize the additive complexities of CFFTs is a special case of the well-known collection-of-sums problem, which is NP-complete [9], [10]. Aiming to reduce additive complexities, previously proposed CSE algorithms focus primarily on identifying recurring subsets of summands (we refer to this as subexpressions or patterns). In contrast, our CSE algorithm, which has only polynomial complexity, also takes advantage of two other types of complexity reductions enabled by the underlying characteristic-2 fields: in addition to explicit recurring subexpressions mentioned above, our CSE algorithm also considers implicit subexpressions for additional savings; since the difference between two sums may require fewer additions than one of the two sums, our CSE algorithm also captures savings of this type.

- We investigate the properties of the three types of CFFTs mentioned above and establish the relations among them. We first show that the three types of CFFTs have the same multiplicative complexities assuming the same bilinear forms. Furthermore, we establish that, under direct implementation, all three types of CFFTs have the same additive complexities. Finally, we show that there is a mapping between SCFFTs and ICFFTs that preserves the additive complexities regardless of implementation. Thus, from the perspective of both multiplicative and additive complexities, SCFFTs and ICFFTs are equivalent. Our results simplify the analysis of their multiplicative and additive complexities as well as performance comparison.

- Using our CSE algorithm, we reduce the additive com- 
plexities of full CFFTs greatly. In comparison to the full CFFTs in [6]-[8], the best results to our knowledge, our CFFTs have 4\%-15\% smaller additive complexities while maintaining the same multiplicative complexities. Compared to some previously proposed FFTs techniques, our CFFTs require fewer multiplications and fewer additions. In comparison to some other FFTs techniques, our CFFTs require fewer multiplications but more additions; in such cases, the total complexities, obtained by assuming that a multiplication over $\operatorname{GF}\left(2^{m}\right)$ is as complex as $2 m-1$ additions, of our CFFTs are smaller.

The rest of the paper is organized as follows. In Section II we briefly review various CFFTs and CSE algorithms to make this paper self-contained. Section III presents our CSE algorithm. We investigate the properties of and relations among the three types of CFFTs in Section IV. CFFTs with reduced additive complexities are obtained by using our CSE algorithm and presented in Section $\mathrm{V}$

\section{BACKGROUND}

\section{A. Cyclotomic FFTs}

Given a primitive element $\alpha \in \mathrm{GF}\left(2^{m}\right)$, the DFT of a vector $\boldsymbol{f}=\left(f_{0}, f_{1}, \ldots, f_{n-1}\right)^{T}$ is defined as $\boldsymbol{F} \triangleq\left(f\left(\alpha^{0}\right), f\left(\alpha^{1}\right), \ldots\right.$ $\left.f\left(\alpha^{n-1}\right)\right)^{T}$, where $f(x) \triangleq \sum_{i=0}^{n-1} f_{i} x^{i} \in \operatorname{GF}\left(2^{m}\right)[x] 1$. A new cyclotomic FFTs algorithm was proposed in [6], and for short lengths (up to 511 [6]) it is computationally efficient. Representing $f(x)$ as a sum of linearized polynomials by cyclotomic decomposition [6], [8], cyclotomic FFT $\boldsymbol{F}=$ $\boldsymbol{A} \boldsymbol{L} \boldsymbol{f}^{\prime}=\boldsymbol{A} \boldsymbol{L} \boldsymbol{\Pi} \boldsymbol{f}$, where $\boldsymbol{A}$ is an $n \times n$ binary matrix, $\boldsymbol{L}=$ $\operatorname{diag}\left(\boldsymbol{L}_{0}, \boldsymbol{L}_{1}, \ldots, \boldsymbol{L}_{l-1}\right)$ is a block diagonal matrix with square matrices $\boldsymbol{L}_{i}$ 's on the diagonal, $l$ is the number of cyclotomic cosets, $\boldsymbol{f}^{\prime}=\left(\boldsymbol{f}_{0}^{\prime T}, \boldsymbol{f}_{1}^{\prime T}, \ldots, \boldsymbol{f}_{l-1}^{\prime T}\right)^{T}$ is a permutation of the input vector $\boldsymbol{f}$, and $\boldsymbol{\Pi}$ is a permutation matrix. Suppose $\boldsymbol{L}_{i}$ corresponds to a coset of size $m_{i}$, using a normal basis of $\operatorname{GF}\left(2^{m_{i}}\right)$ generated by $\gamma_{i}$, then $\boldsymbol{L}_{i}$ becomes a circulant matrix [11]:

$$
\boldsymbol{L}_{i}=\left[\begin{array}{cccc}
\gamma_{i}^{2^{0}} & \gamma_{i}^{2^{1}} & \ldots & \gamma_{i}^{2^{m_{i}-1}} \\
\gamma_{i}^{2^{1}} & \gamma_{i}^{2^{2}} & \ldots & \gamma_{i}^{2^{0}} \\
\vdots & \vdots & \ddots & \vdots \\
\gamma_{i}^{2^{m_{i}-1}} & \gamma_{i}^{2^{0}} & \ldots & \gamma_{i}^{2^{2 m_{i}-2}}
\end{array}\right]
$$

Henceforth in this paper we assume $\boldsymbol{L}_{i}$ 's in $\boldsymbol{L}$ are always constructed by normal bases and we say $\boldsymbol{L}_{i}$ in (1) is a circulant matrix generated by $\gamma_{i}$. Thus the product of $\boldsymbol{L}_{i}$ and $\boldsymbol{f}_{i}^{\prime}$ can be computed as a cyclic convolution, for which fast bilinear form algorithms are available [12]-[15]. These fast algorithms can be written in matrix form as $\boldsymbol{L}_{i} \boldsymbol{f}_{i}^{\prime}=\boldsymbol{Q}_{i}\left(\boldsymbol{R}_{i} \boldsymbol{b}_{i} \cdot \boldsymbol{P}_{i} \boldsymbol{f}_{i}^{\prime}\right)=$ $\boldsymbol{Q}_{i}\left(\boldsymbol{c}_{i} \cdot \boldsymbol{P}_{i} \boldsymbol{f}_{i}^{\prime}\right)$, where $\boldsymbol{b}_{i}=\left(\gamma_{i}, \gamma_{i}^{2}, \ldots, \gamma_{i}^{2^{m_{i}-1}}\right)^{T}, \boldsymbol{Q}_{i}, \boldsymbol{R}_{i}$, and $\boldsymbol{P}_{i}$ are binary matrices, $\boldsymbol{c}_{i}=\boldsymbol{R}_{i} \boldsymbol{b}_{i}$ is a precomputed constant vector, and $\cdot$ stands for pointwise multiplications. Combining all the terms, a DCFFT is given by $\boldsymbol{F}=\boldsymbol{A} \boldsymbol{Q}\left(\boldsymbol{c} \cdot \boldsymbol{P} \boldsymbol{f}^{\prime}\right)$, where $\boldsymbol{Q}$ and $\boldsymbol{P}$ are both block matrices, for which the blocks off the diagonal are the zero matrices and the diagonal blocks are $\boldsymbol{Q}_{i}$ 's and $\boldsymbol{P}_{i}$ 's respectively, and $\boldsymbol{c}=\left(\boldsymbol{c}_{0}^{T}, \boldsymbol{c}_{1}^{T}, \ldots, \boldsymbol{c}_{l-1}^{T}\right)^{T}$. We

\footnotetext{
${ }^{1}$ In this paper, vectors and matrices are represented by boldface letters, and scalars by normal letters.
}

remark that both $\boldsymbol{Q}$ and $\boldsymbol{P}$ are binary and usually sparse. For details of CFFTs, please refer to [6].

Two variants of CFFTs were proposed in [7], [8]. First, by using the same permutation for both $F$ and $f$, SCFFTs proposed in [8] satisfy $\boldsymbol{F}^{\prime}=\boldsymbol{L}^{T} \boldsymbol{A}^{\prime T} \boldsymbol{f}^{\prime}$, where $\boldsymbol{F}^{\prime}=\boldsymbol{\Pi} \boldsymbol{F}$ and $\boldsymbol{f}^{\prime}=\boldsymbol{\Pi} \boldsymbol{f}$. SCFFTs are so named because they have symmetric transform matrices, that is, $\boldsymbol{L}^{T} \boldsymbol{A}^{\prime T}=\boldsymbol{A}^{\prime} \boldsymbol{L}$. It is easy to deduce that $\boldsymbol{A}^{\prime}=\boldsymbol{\Pi} \boldsymbol{A}$. ICFFTs, proposed in [7], are based on inverse DFTs and satisfy $\boldsymbol{F}^{\prime \prime}=\boldsymbol{L}^{-1} \boldsymbol{A}^{-1} \boldsymbol{f}$, where $\boldsymbol{F}^{\prime \prime}$ is also a permutation of $\boldsymbol{F}$. Both SCFFTs and ICFFTs require fewer multiplications than DCFFTs for partial DFTs, where only a subset of components in $\boldsymbol{F}$ are needed.

The multiplicative complexity of each CFFT, i.e., the number of multiplications required, is the total number of nontrivial scalar multiplications in all cyclic convolutions. That is, the multiplicative complexity of $\boldsymbol{c}_{i} \cdot \boldsymbol{P}_{i} \boldsymbol{f}_{i}^{\prime}$ is the number of non-one elements in $\boldsymbol{c}_{i}$ (no element is zero in $\boldsymbol{c}_{i}$ ), which is determined by the cyclic convolution algorithms. To find the optimum cyclic convolution algorithms with the minimum multiplicative complexities in CFFTs is still an open problem. In this paper, we use the cyclic convolution algorithms in [16].

The additive complexity of each CFFT is determined by the two matrix-vector multiplications in which both matrices are binary. For example, in DCFFTs, the matrices are $A Q$ and $\boldsymbol{P}$. Due to the large size of $\boldsymbol{A Q}$, direct computation of the matrix-vector product will result in high additive complexity. A heuristic algorithm based on erasure decoding [17] was used in [6] to reduce the additive complexity. Similar optimization was also used in [7]. Another fast matrix-vector multiplication algorithm is the Four Russians' algorithm [18], but it is based on preprocessing and fails to efficiently exploit the matrix structure. CSE is another commonly used technique for fast matrix-vector multiplication.

\section{B. Common Subexpression Elimination}

Consider a linear transform $\boldsymbol{Y}=\boldsymbol{M} \boldsymbol{X}$, where $\boldsymbol{Y}$ and $\boldsymbol{X}$ are $n$ - and $n^{\prime}$-dimensional column vectors, respectively, and $M$ is an $n \times n^{\prime}$ matrix containing only $1,-1$, and 0 . Clearly, such a transform requires only additions and subtractions. It was shown that it is an NP-complete problem [9, Ensemble Computation], [10, Collection of Sums] to minimize the number of additions and subtractions.

A special type of the collection-of-sums problem is the MCM problem [19], where the relative position of a bit pattern within the matrix is of no importance [20]. This is a valid assumption in the case of the $\boldsymbol{X}=\left(c^{0} x_{0}, c^{1} x_{0}, \ldots, c^{n-1} x_{0}\right)^{T}$ with $c=2$ or $c=2^{-1}$, which is common in filters. Thus, patterns that differ in relative positions only can be obtained from one of them by shift operations. This class of problems have wide applications in finite impulse response (FIR) filters [19]-[25]. Graph-based algorithms [24], [25] synthesize directed acyclic graphs, in which partial sums define nodes and shifts are annotated on edges. In [24], optimal solutions can be obtained by exhaustive search of all topologies with high computational complexity. Entropy and conditional entropy are used in [25] for vertex decomposition. Pattern-based algorithms [19]-[23], [26], [27] reduce the MCM complexity by 
first identifying recurring patterns, which are combinations of non-zero positions, and then calculating them only once. They usually use canonical signed digit (CSD) representation to identify potentially sharable subexpressions. The algorithms in [19], [21]-[23], [26], [27] use weight-2 subexpressions as the primitive elements. In contrast, [20] searches for the highestweight common subexpressions.

To minimize the number of additions in the matrix-vector multiplications in CFFTs over characteristic-2 fields constitutes a different type of the collection-of-sums problem, where $\boldsymbol{X}$ is over characteristic-2 fields. This implies two properties: (1) the summands are independent, and (2) the 1's in $M$ are equivalent to -1 and additions are equivalent to subtractions. The second property is noted but not utilized in [19]. Due to the two properties, CSE techniques for the MCM problem are not suitable for the problem considered in this paper. Heuristic CSE techniques proposed in the context of MCM problem result in modest complexity reductions since they do not take advantage of the second property (see, for example, [19]). For the first property, sophisticated CSE techniques, especially those relying on CSD representation, that are tailored for FIR filters (see, for example, [20]-[25]) cannot be directly applied to our problem; to adapt these algorithms to our problem is not straightforward and requires nontrivial research efforts. Hence, we do not consider CSE techniques for the MCM problem in this paper.

Two CSE algorithms that account for the cancelation property were proposed in [6], [28], and we will compare our CSE algorithms against these.

\section{A Novel CSE Algorithm Over CHARACTERISTIC-2 FIELDS}

We propose a novel CSE algorithm with polynomial complexity that significantly reduces the additive complexities of CFFTs. Although our CSE algorithm does not guarantee to minimize the additive complexities, it may do so in some cases, especially when the size of the problem is small.

Let us establish the terminology to describe our CSE algorithms. For a matrix-vector multiplication $\boldsymbol{Y}=\boldsymbol{M X}$, where $\boldsymbol{Y}=\left(Y_{0}, Y_{1}, \ldots, Y_{n-1}\right)^{T}$ and $\boldsymbol{X}=\left(X_{0}, X_{1}, \ldots, X_{n^{\prime}-1}\right)^{T}$ are $n$ - and $n^{\prime}$-dimensional column vectors and $M$ is an $n \times n^{\prime}$ matrix, we refer to the components in $\boldsymbol{Y}$ as sums and the components in $\boldsymbol{X}$ as summands. Note that the sums in $\boldsymbol{Y}$ have one-to-one correspondence with the rows in $M$, and in direct computation the number of additions required to compute a sum is the number of ones in its corresponding row minus one. Hence, with a slight abuse of terminology, we sometimes use rows and sums in an exchangeable manner. Similarly, there is a one-to-one correspondence between the summands and the columns in $\boldsymbol{M}$, and we sometimes use columns and summands in an exchangeable fashion below.

Our CSE algorithm achieves two kinds of savings: differential savings and recurrence savings, as defined in Sections III-A and ЩI-B, respectively.

\section{A. Differential Savings}

Let $\boldsymbol{Y}=\boldsymbol{M} \boldsymbol{X}$ represent a matrix-vector multiplication, in which $\boldsymbol{X}$ is over characteristic-2 fields and $\boldsymbol{M}$ is a binary matrix. For the column positions where $M_{r_{p}}$ and $M_{r_{c}}$, rows $r_{p}$ and $r_{c}\left(r_{p} \neq r_{c}\right)$ of $\boldsymbol{M}$ respectively, both have ones, the difference (or sum) $\boldsymbol{M}_{r_{c}}-\boldsymbol{M}_{r_{p}}$ of the two rows has zeros. If $M_{r_{c}}-M_{r_{p}}$ contains fewer entries than one of the two rows, say $\boldsymbol{M}_{r_{c}}$, we can reduce the total number of additions by first computing $Y_{r_{p}}$ and then computing $Y_{r_{c}}=Y_{r_{p}}+\left(\boldsymbol{M}_{r_{c}}-\right.$ $\left.\boldsymbol{M}_{r_{p}}\right) \boldsymbol{X}$. Let us denote the numbers of non-zero entries in $\boldsymbol{M}_{r_{p}}, \boldsymbol{M}_{r_{c}}$, and $\boldsymbol{M}_{r_{c}}-\boldsymbol{M}_{r_{p}}$ as $w_{p}, w_{c}$, and $w_{d}$, respectively, the differential saving (the number of additions saved) is given by $w_{c}-w_{d}-1$. Since we are only concerned about positive savings, we use $\left(w_{c}-w_{d}-1\right)^{+} \triangleq \max \left\{0, w_{c}-w_{d}-1\right\}$ in our algorithms.

The price for the differential saving is that now $Y_{r_{p}}$ must be computed before $Y_{r_{c}}$, putting a dependency between the two sums. We use an ordered pair $\left(r_{p}, r_{c}\right)$ to represent this dependency; we call $Y_{r_{p}}$, the sum computed first, the parent, and refer to $Y_{r_{c}}$ as the child. Since each ordered pair introduces a dependency, to keep track of all dependency, we use a digraph to keep track of all ordered pairs, where the vertices are the row numbers in the ordered pairs and the edges are from the parent to the child in each pair. We call this graph dependency graph henceforth in this paper. There is no conflicting dependency as long as the dependency graph is acyclic. Thus, before any ordered pair can be added to the dependency graph, it is necessary to check whether the addition of the new ordered pair will introduce cycles in the dependency graph; if yes, this ordered pair is called cycleinducing and hence not permissible. Cycle detection can be done recursively.

When an ordered pair $\left(r_{p}, r_{c}\right)$ is added to the dependency graph, both $\boldsymbol{M}$ and $\boldsymbol{X}$ need to be transformed. We first append $Y_{r_{p}}$ to $\boldsymbol{X}$ as a new summand. We also replace $\boldsymbol{M}_{r_{c}}$ with the difference $\boldsymbol{M}_{r_{c}}-\boldsymbol{M}_{r_{p}}$; then due to the new summand $Y_{r_{p}}$, a new column with a single one at the $r_{c}$ th position and zeros at other positions is appended to $\boldsymbol{M}$. We call these operations a differential transformation.

Our differential transformations bear some similarities to the erasure correction approach used in [17]. As pointed out in [17], $\boldsymbol{Y}=\boldsymbol{M} \boldsymbol{X}$ is equivalent to $[\boldsymbol{M} \mid \boldsymbol{I}]\left(\boldsymbol{X}^{T}, \boldsymbol{Y}^{T}\right)^{T}=\mathbf{0}$, which defines a code $\mathcal{C}$ with all codewords $\left(\boldsymbol{X}^{T}, \boldsymbol{Y}^{T}\right)^{T}$; to compute $\boldsymbol{Y}=\boldsymbol{M} \boldsymbol{X}$ is equivalent to erasure correction with $\boldsymbol{Y}$ erased based on $\mathcal{C}$. After a series of differential transformations as described above, the matrix-vector multiplication becomes $\boldsymbol{Y}=\boldsymbol{M}^{\prime} \boldsymbol{X}^{\prime}$, where $\boldsymbol{X}^{\prime}=\left(\boldsymbol{X}^{T}, \boldsymbol{Y}^{\prime T}\right)^{T}, \boldsymbol{Y}^{\prime}$ consists of the summands corresponding to all the parents in the ordered pairs, and $M^{\prime}$ has the same number of rows as $M$. By adding all-zero columns to $M^{\prime}$, we can find a matrix $M^{\prime \prime}$ such that $\boldsymbol{Y}=\boldsymbol{M}^{\prime \prime}\left(\boldsymbol{X}^{T}, \boldsymbol{Y}^{T}\right)^{T}$. Hence $\left(\boldsymbol{M}^{\prime \prime}-[\mathbf{0} \mid \boldsymbol{I}]\right)\left(\boldsymbol{X}^{T}, \boldsymbol{Y}^{T}\right)^{T}=$ 0. Thus our differential transformations lead to a different parity check matrix for the same code $\mathcal{C}$. Furthermore, the acyclic property for the dependency graph ensures that $\boldsymbol{Y}$ can be recovered by using the parity check matrix $\boldsymbol{M}^{\prime \prime}-[\mathbf{0} \mid \boldsymbol{I}]$. From this perspective our differential transformation is similar to that of the message passing part of [17]: both find an alternative parity check matrix with smaller Hamming weights for the code $\mathcal{C}$, which can be used to compute $\boldsymbol{Y}$. However, different search methods are used to obtain alternative parity check matrices in our work and in [17]. 


\section{B. Recurrence Savings}

We refer to the number of occurrences of a subexpression (or pattern in the rows of $\boldsymbol{M}$ ) as pattern frequency, and define the recurrence saving of each pattern as its pattern frequency minus 1. After a subexpression is identified, we append the subexpression to $\boldsymbol{X}$ as a new summand, and $\boldsymbol{M}$ is updated accordingly. These operations are referred to as a recurrence transformation. A sequence of recurrence transformations can be described in a matrix decomposition form: $\boldsymbol{M}=\boldsymbol{M}_{R} \prod_{i=0}^{K-1} \boldsymbol{T}_{i}$, where $\boldsymbol{T}_{i}=\left[\boldsymbol{I} \mid \boldsymbol{G}_{i}^{T}\right]^{T}$, the row vector $\boldsymbol{G}_{i}$ corresponds to a subexpression, $\boldsymbol{M}_{R}$ has no pattern recurrence, and $K$ is the number of identified subexpressions. Thus $\boldsymbol{Y}$ is computed in a sequential fashion: first assign $\boldsymbol{X}^{(0)}=\boldsymbol{X}$, then compute $\boldsymbol{X}^{(i+1)}=\boldsymbol{T}_{i} \boldsymbol{X}^{(i)}$ for $i=0,1, \ldots, K-1$, and finally compute $\boldsymbol{Y}=\boldsymbol{M}_{R} \boldsymbol{X}^{(K)}$. For $0 \leq i \leq K-1$, let $\boldsymbol{M}^{(i)}$ denote $\boldsymbol{M}_{R} \prod_{l=i}^{K-1} \boldsymbol{T}_{l}$, and $\boldsymbol{Y}=\boldsymbol{M}^{(i)} \overline{\boldsymbol{X}}^{(i)}$.

Compared with the matrix splitting method [20], recurrence transformations keep track of the identified subexpressions as new summands, instead of simply removing them. To reduce the computational complexity of pattern search, our CSE algorithm looks for only two-summand subexpressions. However, since each two-summand subexpression is in turn appended as a summand and multi-summand subexpressions can be expressed recursively as two-summand subexpressions, our CSE algorithm efficiently exploits the recurrence savings of both two-summand patterns and multi-summand patterns.

One limitation of the recurrence transformations above is that it considers only explicit subexpressions, missing implicit subexpressions that are hidden by cancelation. We will now identify implicit subexpressions through forced patterns. To this end, after a two-summand pattern $X_{0}+X_{1}$ is identified and introduced as a new summand $X_{n}$, we try to impose the pattern on the rows containing only $X_{0}$ or $X_{1}$ by replacing $X_{0}$ with $X_{1}+X_{n}$ or $X_{1}$ with $X_{0}+X_{n}$. After forcing patterns $X_{1}+X_{n}$ or $X_{0}+X_{n}$ on row $r_{i}$, if previously identified patterns emerge due to cancelation and therefore lead to complexity savings, we transform $M_{r_{i}}$ to reflect the forced pattern. If the forced pattern does not lead to any saving, we do not transform $M_{r_{i}}$. Since a forced pattern leads to complexity saving only when they match previously identified patterns, we search the rows only for previously identified patterns. Since we keep track of all two-summand patterns, we first search the rows for previously identified patterns that include $X_{0}$ or $X_{1}$, which is inserted due to the forced pattern. If we find a previously identified pattern, say $X_{j}=X_{i}+X_{0}$, in row $r_{i}$, we replace $X_{i}+X_{0}$ by $X_{j}$ and continue to search for all previously identified patterns that include $X_{j}$, and so on.

Now we illustrate the advantage of the forced pattern method by a simple example. Say we have established three patterns as $X_{4}=X_{1}+X_{2}, X_{5}=X_{3}+X_{4}$, and $X_{6}=X_{0}+X_{1}$. Now let us consider the sum $Y_{0}=X_{0}+X_{2}+X_{3}$, which does not contain the identified patterns $X_{4}, X_{5}$, or $X_{6}$ explicitly. But if we force $X_{6}$ on $Y_{0}$, we have $Y_{0}=X_{1}+X_{2}+X_{3}+X_{6}$, which becomes $Y_{0}=X_{5}+X_{6}$ after replacing previously identified patterns $X_{1}+X_{2}$ with $X_{4}$ and $X_{3}+X_{4}$ with $X_{5}$ as described above. In this simple example, by forcing the pattern we reduce the number of additions by one. In a nutshell, it is a greedy strategy in which, based on existing subexpressions, we try to find an alternative expression that requires fewer additions for a sum.

When introducing forced patterns for a sum, new summands for the sum are introduced. If any new summand is a sum, this introduces dependency between the two sums, and possibly cycles in the dependency graph. We replace $X_{1}$ with $Y_{1}$ in the simple example above to illustrate such a case. If we force the pattern $X_{6}=X_{0}+Y_{1}$ pattern on $Y_{0}$, we have $Y_{0}=$ $Y_{1}+X_{2}+X_{3}+X_{6}=X_{5}+X_{6}$. Although it reduces the number of additions by one, it requires that $Y_{1}$ should be computed before $Y_{0}$. Since forced patterns introduce new dependency, we will keep track of this using the dependency graph and cycle detection is necessary in recurrence transformations if we consider forced patterns.

\section{Approximate Dynamic Programming}

We have discussed two kinds of transformations that result in differential savings and recurrence savings. A remaining question is: how should we coordinate the transformations associated with differential savings and recurrence savings? That is, which kind of saving is more preferable? A seemingly straightforward answer would be to use a simple greedy strategy: choose one transformation with the greatest saving. Instead of this simple greedy strategy, we adopt a different strategy. We justify our choice by approximate dynamic programming [29] below.

Note that both differential and recurrence transformations can be expressed in a matrix decomposition form. Thus the collection-of-sums problem can be viewed as a dynamic programming problem [29], where the cost to be minimized is the number of additions and each differential or recurrence transformation corresponds to one stage. The total cost is denoted by $A=\sum_{i=0}^{K-1} g^{(i)}+J_{R}$ where $g^{(i)} \in\{0,1\}$ is the cost of Stage $i$ and $J_{R}$ is the cost of implementing $M_{R}$. Let us denote $\boldsymbol{M}$ and $\boldsymbol{X}$ after the $i$ th stage as the $\boldsymbol{M}^{(i)}$ and $\boldsymbol{X}^{(i)}$, and they are the state variables. The idea of approximate dynamic programming is to approximate and optimize the cost-to-go $J$ [29]. Suppose after the transformations in Stage $i$, the matrix-vector multiplication is given by $\boldsymbol{Y}=\boldsymbol{M}^{(i)} \boldsymbol{X}^{(i)}$. Since under direct computation, it needs $W\left(\boldsymbol{M}^{(i)}\right)-n$ additions, where $W\left(\boldsymbol{M}^{(i)}\right)$ is the number of 1's in $\boldsymbol{M}^{(i)}$, we use $J^{(i)}=a^{(i)}\left(W\left(\boldsymbol{M}^{(i)}\right)-n\right)$ as a linear approximation of the cost-to-go, where $a^{(i)}$ approximates $\left(A-\sum_{j=0}^{i-1} g^{(j)}\right) /\left(W\left(M^{(i)}\right)-n\right)$. When $a^{(i)}=(A-$ $\left.\sum_{j=0}^{i-1} g^{(j)}\right) /\left(W\left(\boldsymbol{M}^{(i)}\right)-n\right), J^{(i)}=A-\sum_{j=0}^{i-1} g^{(j)}$ is indeed the cost-to-go. Suppose for Stage $i$, the largest differential and recurrence savings are $s_{d}^{(i)}$ and $s_{r}^{(i)}$, respectively. Based on the above approximation, we can find a transformation that minimizes the cost-to-go. If a differential transformation is chosen, the matrix weight after the transformation is given by $W\left(\boldsymbol{M}^{(i+1)}\right)=W\left(\boldsymbol{M}^{(i)}\right)-s_{d}^{(i)}$; otherwise, it is $W\left(\boldsymbol{M}^{(i+1)}\right)=W\left(\boldsymbol{M}^{(i)}\right)-s_{r}^{(i)}-1$. Then the approximate optimal cost-to-go is the smaller between $a^{(i)} \cdot\left(W\left(M^{(i)}\right)-\right.$ $\left.s_{d}^{(i)}-n\right)$ and $1+a^{(i)} \cdot\left(W\left(M^{(i)}\right)-s_{r}^{(i)}-1-n\right)$. Thus a differential transformation is preferred when $s_{d}^{(i)}>s_{r}^{(i)}+$ $1-1 / a^{(i)}$, and a recurrence transformation is preferred if 
$s_{d}^{(i)} \leq s_{r}^{(i)}+1-1 / a^{(i)}$. Although it is difficult to compute $a^{(i)}$ since $A$ is actually unknown, fortunately the choice between differential saving and recurrence saving does not require the precise value of $A$. It is obvious that $0<a^{(i)}<1$ for any $i$, and hence differential transformations are usually preferred over recurrence transformations even when $s_{d}^{(i)}=s_{r}^{(i)}$. This is particularly the case when $a^{(i)}$ is small. For example, the ratio of the required number of additions after applying our CSE algorithm, and $W\left(M^{(0)}\right)-n$ is between 0.16 and 0.26 . Thus, $a^{(0)}$ is clearly a small fraction. As $i$ increases, $a^{(i)}$ increases while $s_{d}^{(i)}$ and $s_{r}^{(i)}$ decrease. Our CSE algorithm treats the differential transformations with preference in all cases.

We comment that the simple greedy strategy which selects the greater one between $s_{d}^{(i)}$ and $s_{r}^{(i)}$ mentioned above corresponds to always setting $a^{(i)}=1$ in approximate dynamic programming, which does not provide a good approximation. Our simulation results confirm this observation, as the differential saving first strategy usually leads to better results than the simple greedy strategy.

Since we are using approximate dynamic programming in every stage, choosing a differential saving does not take into account all recurrence savings in future stages. Thus for some stages it may be an unwise choice. We propose a method to identify such differential savings and reverse them. Say $Y_{0}=$ $X_{0}+X_{1}+X_{2}+X_{3}$ and $Y_{1}=X_{0}+X_{4}+X_{5}+Y_{0}$ as a result of the differential saving from an ordered pair $(0,1)$. Since $Y_{0}+X_{0}=X_{1}+X_{2}+X_{3}$, we can replace $X_{0}+Y_{0}$ in $Y_{1}$ by $X_{1}+X_{2}+X_{3}$ and it is clear that $Y_{0}$ and $Y_{1}$ have a common subexpression $X_{1}+X_{2}+X_{3}$. Using the subexpression $X_{1}+$ $X_{2}+X_{3}$ effectively reverses the differential transformation represented by $(0,1)$. To identify a reversal of this kind, we search for reversal patterns; a reversal pattern $Y_{i}+X_{j}$ consists of a sum $Y_{i}$ and one of its summands $X_{j}$. In contrast to other patterns, this pattern may have a recurrence saving of zero, that is, it appears only once. It can be shown that such a reversal saves only one addition, regardless of the frequency of the reversal pattern; thus, such a reversal is meaningful only when there are no other subexpressions involving $Y_{i}$. For instance, in the above example, if there are more than two recurrences of $Y_{0}+X_{4}$, the subexpression $Y_{0}+X_{4}$ results in a greater saving than $Y_{0}+X_{0}$. Thus it will be efficient to search for reversal patterns only after all recurrence savings are accounted for.

Our CSE algorithm, shown below in Algorithm 1, has two major steps, Steps 11 and 1 3, and they are referred to as the differential and recurrence steps respectively.

\section{Algorithm 1. Common Subexpression Elimination}

11 Identify the non-cycle-inducing pairs of rows with the $l_{d}$ greatest differential savings, select one pair out of them randomly, and transform both $\boldsymbol{M}$ and $\boldsymbol{X}$ as described above.

12 Repeat Step 111 until there is no differential saving.

13 Identify the two-summand patterns with the $l_{r}$ greatest recurrence savings, select one out of them randomly. Replace all occurrences of the selected pattern with a new entry. On those rows with only one entry of the pattern, force the pattern if it leads to less 1's in the row.

1.4 Go to Step 11 until there is no recurrence saving.

1.5 If there is a reversal pattern, reverse the differential saving and go to Step 13.

Since differential savings are due to the overlapping ones in two rows, there is no positive differential saving if there is no recurrence saving. This is the reason for the termination condition in Step 114. In Steps 11 1 and 113, we randomly select one transformation among those with the $l_{d}$ greatest differential savings and the $l_{r}$ greatest recurrence savings respectively. There is a tradeoff between search space (and hence performance) and search complexity: greater $l_{d}$ and $l_{r}$ enlarge the search space that may lead to greater savings at the expense of higher complexity. In our work, $l_{d}=l_{r}=2$ appears enough for most cases. For matrices with small sizes, the additional complexity caused by expanding the searching space is usually affordable. For large matrices, we use $l_{d}=l_{r}=1$. Since Algorithm 1 is a randomized algorithm, the result of each run may vary. However, simulation results show that the variance between different runs is relatively small in comparison to the number of required additions.

Our sequential transformation of $M$ in Section $\amalg-\mathrm{B}$ is similar to the CSE algorithm in [26], but the algorithm in [26] does not take advantage of the cancelation property of characteristic2 fields as Algorithm 11. With forced patterns, Algorithm 1 takes advantage of the cancelation property not only by differential savings but also by recurrence savings. Although Algorithm 1 and those in [17], [28] all take advantage of the cancelation property of characteristic- 2 fields, they use quite different strategies. Algorithm 1 uses a top-down approach to build the addition sequence by reducing the binary matrix, while a bottom-up approach starting from summands was used in [28]. The CSE algorithm in [17] first rebuilds the binary matrix from low-weight linear combinations of rows, then reduces the matrix top-down using recurrence savings. Also, although the method in [17] takes advantage of the cancelation property by erasure decoding in the message passing part, it fails to do so in its CSE part. As we will show in Section V Algorithm 1 leads to significantly better results than the method in [17].

\section{Fast CSE}

When the size of $M$ is large, the time complexity of Algorithm 1 may be prohibitive. We propose several improvements to reduce the time complexity of Algorithm 1

In Algorithm 1, we restart the differential step after each recurrence step. But the possibility that new differential savings emerge after we identify a pattern for recurrence saving is quite small. In order to reduce the complexity, we do not revisit the differential step after the recurrence step has ended, essentially decoupling the two steps. This not only reduces the time complexity by reducing the number of times Step 1.1 is repeated, but also enables us to further accelerate both steps by space-time tradeoff, which will be discussed below. Note that our simulation results show that the decoupling of the two steps results in only negligible performance loss.

Now that the differential step is standalone, it is necessary to avoid repeated exhaustive searches. There are only $n$ rows 
in $M$, so all possible differential saving can be put in an $n \times n$ array $\boldsymbol{D}$, where $D_{i j}$ stands for the differential saving of the ordered pair of rows $\left(r_{i}, r_{j}\right)$. An exhaustive search is needed to initialize $\boldsymbol{D}$. Afterwards, at most $2(n-1)$ entries (namely, the non-diagonal entries in row $r_{i}$ and column $r_{j}$ ) of the array need to be updated after each ordered pair $\left(r_{i}, r_{j}\right)$ is added to the dependency graph. Whenever one pair of rows is detected to be cycle-inducing, its differential saving will be set to -1 and hence it is excluded from future consideration for differential transformations. As the number of possible pairs decreases continuously, the search will be increasingly simpler.

A similar idea can be used to reduce the time complexity of the recurrence step. Since elimination of one pattern will only change a small portion of the pattern frequencies, to expedite searches, we store the recurrence savings and update them after each recurrence transformation.

Because not all patterns exist and the number of possible patterns will decrease continuously, it will require less storage space if we keep track of only the patterns with positive recurrence savings. However, this will involve an exhaustive search to update the pattern frequencies each time after a pattern is identified, which may results in high time complexity when the size of $M$ is large. Instead, we keep track of all pattern frequencies, including those with no recurrence savings, in a two-dimensional array $\boldsymbol{R}$. Suppose after the differential steps are over, and $\boldsymbol{M}^{\prime}$ has $\bar{n}$ columns. Initially, $\boldsymbol{R}$ is an upper triangle array with $\bar{n}-1$ rows, where $R_{i j}$ is the recurrence saving of the two-summand pattern $X_{i}+X_{i+j+1}$ for $0 \leq i \leq \bar{n}-2$ and $0 \leq j \leq \bar{n}-i-2$. The recurrence saving array $\boldsymbol{R}$ is arranged in this fashion so that frequency updates can be done by direct addressing without search and it is not necessary to remove frequencies. When a new pattern is identified, the twosummand pattern becomes the $(\bar{n}+1)$ th summand. Thus, the frequency of the $i$ th and the $(\bar{n}+1)$ th summands is appended to the $i$ th row. Furthermore, a new row with only one element, the frequency of the $\bar{n}$ th and $(\bar{n}+1)$ th summands, will be the last row of $\boldsymbol{R}$. After $X_{i}+X_{j}$ is identified as a subexpression, all frequencies related to $X_{i}$ or $X_{j}$ need to be updated. That is, $R_{i^{\prime}, i-i^{\prime}-1}$ for all $i^{\prime}<i, R_{j^{\prime}, j-j^{\prime}-1}$ for all $j^{\prime}<j, R_{i, i^{\prime \prime}-i-1}$ for all $i^{\prime \prime}>i$, and $R_{j, j^{\prime \prime}-j-1}$ for all $j^{\prime \prime}>j$ are updated accordingly. Furthermore, $R_{i, j-i-1}$ is set to zero.

During Step 11, our CSE algorithm keeps only one copy of each row. Actually one row can have different decompositions, based on differential savings with different rows. To exploit the best differential saving for each row, a modified differential saving update scheme is developed.

Let us assume that the ordered pair $\left(r_{p}, r_{c}\right)$ is selected for differential transformation, which replaces $\boldsymbol{M}_{r_{c}}$ with $\boldsymbol{M}_{r_{c}}^{\prime}$. For row $r_{i}\left(r_{i} \neq r_{p}\right)$, there are two possible differential savings: one between $M_{r_{c}}$ and $\boldsymbol{M}_{r_{i}}$ and the other between $\boldsymbol{M}_{r_{c}}^{\prime}$ and $M_{r_{i}}^{\prime}$. If the latter is greater, we simply update $D_{c i}$. If the former is greater, the differential saving $D_{c i}$ is not changed and $M_{r_{c}}$ is saved so that it can be used when $D_{c i}$ is selected. If two savings are equal, it is randomly chosen which copy to use. Since we may need different copies of $\boldsymbol{M}_{r_{c}}$ for each $r_{i}$, a three-dimension array $\boldsymbol{K}$ whose entry $K_{i j}$ keeps a copy of $\boldsymbol{M}_{r_{j}}$ corresponding to $D_{i j}$ if $\boldsymbol{M}_{r_{j}}$ provides a greater differential saving than $M_{r_{j}}^{\prime}$ with regard to $M_{r_{i}}$. Since this can occur recursively, for each row at most $n-1$ different rows may be stored in $\boldsymbol{K}$.

Our CSE algorithm incorporating the above improvements is shown in Algorithm 2

\section{Algorithm 2. Fast CSE}

2.1 Initialize the differential saving array $\boldsymbol{D}$ and $\boldsymbol{K}$.

[2. 2 Find the non-cycle-inducing pairs of rows with the $l_{d}$ greatest differential savings in $D$, randomly choose one, eliminate it, and update $\boldsymbol{D}$ and $\boldsymbol{K}$ accordingly.

2.3 Repeat Step 2/2 until there is no positive entry in $\boldsymbol{D}$.

2. 4 Initialize the recurrence saving array $\boldsymbol{R}$.

225 Find the patterns with the $l_{r}$ greatest recurrence savings in $\boldsymbol{R}$, randomly choose one, replace all occurrences of it. On those rows with only one entry of the pattern, force the pattern if it leads to less 1's in the row. Update $\boldsymbol{R}$.

2.6 Repeat Step 2.5 until all entries in $\boldsymbol{R}$ are zero.

[2. 7 If there is a reversal pattern, reverse the differential saving, update $\boldsymbol{R}$, and go to Step 2$] 5$.

Our simulation results show that after a single run, the difference between the total additive complexities obtained by Algorithms 1 and 2 is negligible. However, the time complexity of Algorithm 2 is much smaller than that of Algorithm 1. For example, when $M$ is a $255 \times 255$ matrix, for a single run Algorithm 1 needs about ten hours while Algorithm 2 finishes in approximately five minutes. The difference in run time is greater for matrices with larger sizes. Since Algorithms 1 and 2 are both probabilistic, the speed advantage of Algorithm 2 over Algorithm 1 enables us to run Algorithm 2 many more times, enhancing the possibility of obtaining a better result than using Algorithm 1 within the same amount of time.

\section{E. Example}

Now we provide an example of Algorithm 2, At the beginning, $\boldsymbol{K}$ is empty and

$$
\boldsymbol{M}=\left[\begin{array}{lllll}
1 & 0 & 1 & 1 & 1 \\
1 & 1 & 1 & 1 & 1 \\
1 & 1 & 0 & 1 & 1 \\
0 & 1 & 1 & 1 & 0
\end{array}\right] \quad \boldsymbol{D}=\left[\begin{array}{cccc}
-1 & 3 & 1 & 0 \\
2 & -1 & 2 & 0 \\
1 & 3 & -1 & 0 \\
0 & 2 & 0 & -1
\end{array}\right]
$$

Choosing $(0,1)$ and adding a column corresponding the new summand $Y_{0}$, we have

$$
\boldsymbol{M}^{(1)}=\left[\begin{array}{llllll}
1 & 0 & 1 & 1 & 1 & 0 \\
0 & 1 & 0 & 0 & 0 & 1 \\
1 & 1 & 0 & 1 & 1 & 0 \\
0 & 1 & 1 & 1 & 0 & 0
\end{array}\right] \quad \boldsymbol{D}=\left[\begin{array}{cccc}
-1 & -1 & 1 & 0 \\
-1 & -1 & 2 & 0 \\
1 & 0 & -1 & 0 \\
0 & 0 & 0 & -1
\end{array}\right] \text {. }
$$

Since $(1,0)$ is cycle-inducing, its saving is simply set to -1 . We also set $K_{12}$ to $(1,1,1,1,1)$ to keep track of $\boldsymbol{M}_{1}$.

Choosing $(1,2)$, the matrices are updated as

$$
\begin{aligned}
\boldsymbol{M}^{(2)} & =\left[\begin{array}{ccccccc}
1 & 0 & 1 & 1 & 1 & 0 & 0 \\
0 & 1 & 0 & 0 & 0 & 1 & 0 \\
0 & 0 & 1 & 0 & 0 & 0 & 1 \\
0 & 1 & 1 & 1 & 0 & 0 & 0
\end{array}\right] \\
\boldsymbol{D} & =\left[\begin{array}{cccc}
-1 & -1 & 0 & 0 \\
-1 & -1 & -1 & 0 \\
-1 & 0 & -1 & 0 \\
0 & 0 & 0 & -1
\end{array}\right]
\end{aligned}
$$


Note that $(2,0)$ is cycle-inducing so there is no positive differential saving left.

Now we enter the recurrence transformations. The recurrence saving array $\boldsymbol{R}$ for $\boldsymbol{M}^{(2)}$ is initialized to all zeros except that $R_{2,0}=1$, which corresponds to the pattern $X_{2}+X_{3}$. Hence $\boldsymbol{G}_{0}$ is $(0,0,1,1,0,0,0)$ and the algorithm stops at

$$
\boldsymbol{M}_{R}=\left[\begin{array}{llllllll}
1 & 0 & 0 & 0 & 1 & 0 & 0 & 1 \\
0 & 1 & 0 & 0 & 0 & 1 & 0 & 0 \\
0 & 0 & 1 & 0 & 0 & 0 & 1 & 0 \\
0 & 1 & 0 & 0 & 0 & 0 & 0 & 1
\end{array}\right]
$$

and the recurrence saving array becomes all zeros. The remaining matrix $M_{R}$ needs five additions. The identified pattern $X_{2}+X_{3}$ also needs one addition. So $\boldsymbol{Y}=\boldsymbol{M X}$ can be calculated by six additions, whereas a straightforward implementation of $\boldsymbol{Y}=\boldsymbol{M} \boldsymbol{X}$ requires 12 additions. Note that techniques such as forced patterns or reversal patterns are not applicable in this simple example. Nevertheless, since it can be easily verified that $\boldsymbol{Y}=\boldsymbol{M} \boldsymbol{X}$ cannot be done in five additions, our CSE algorithm minimizes the number of additions in this case. Note that if we only use recurrence savings, the result will be seven additions.

\section{F. Time and Storage Complexities}

Since the reduction of additive complexities depends on $M$ only, the output of Algorithm 2 for a given CFFT can be used for any input vector. Hence Algorithm 2 is simply precomputation and its complexity should not be considered as part of the complexities of CFFTs. To show that Algorithm 2 is computationally tractable, we provide an order-of-magnitude analysis for the time and area complexity of Algorithm 2 below.

Algorithm 2 requires only four types of operations: adding two rows, inserting or removing entries from a row, searching for a two-summand pattern in a row, and comparison to find the greatest saving. During the optimization, while the number of columns in the matrix $\boldsymbol{M}^{(i)}$ increases continuously, the number of 1's in each row decreases. To facilitate row additions, for each row we only store the positions of 1's as a sorted list. Since the original $M$ has $n^{\prime}$ columns, adding two rows is equivalent to merging two sorted lists of size at most $n^{\prime}$, which requires at most $2 n^{\prime}$ comparisons. For simplicity, we assume inserting or removing entries in a row has the same complexity as adding two rows. Searching for a two-summand pattern in a row needs at most $n^{\prime}$ comparisons. We assume the complexity of either appending an entry to a row or updating a matrix entry is negligible.

Now since differential transformations described in Steps 2 1, 22 2, and 2 3 and recurrence transformations in Steps 2] 4, 2], and 26 are independent, we can analyze them separately. In Step 21 , the initialization of the differential saving array $\boldsymbol{D}$ needs to add rows for $n(n-1) / 2$ times, so it takes at most $n(n-1) n^{\prime}$ comparisons. The result of Step 22 is an acyclic digraph with at most $n$ nodes, so at most $n(n-1) / 2$ pairs of rows are identified. To identify one pair of rows, we need at most $g-1$ comparisons, where $g$ is the number of remaining pairs of rows. After one differential saving is identified, the child row needs to be updated, which requires at most $2 n^{\prime}$ comparisons. Correspondingly, computing the differential savings relative to the new child row needs up to $2 n^{\prime}(n-2)$ comparisons since the parent row is ineligible. So it will take at most $\sum_{g=n(n-1) / 2}^{1}\left(2 n^{\prime}(n-2)+2 n^{\prime}+g-1\right) \approx O\left(n^{4}+n^{2} n^{\prime}\right)$ comparisons. Updating $\boldsymbol{K}$ does not requires extra computation. Therefore the number of total comparisons for differential transformations is $O\left(n^{4}+n^{2} n^{\prime}\right)$.

To initialize $\boldsymbol{R}$, we scan the matrix row by row to find the recurrences of each two-summand pattern. For any row, we increase $R_{i j}$ by one if the two-summand pattern $X_{i}+X_{i+j-1}$ is present. Since there are at most $n^{\prime} 1$ 's in a row, it has at most $n^{\prime}\left(n^{\prime}-1\right) / 2$ two-summand patterns and hence requires at most $n^{\prime}\left(n^{\prime}-1\right) / 2$ additions. Thus Step 24 needs at most $n n^{\prime}\left(n^{\prime}-1\right) / 2$ additions. For the first recurrence transformation, it will need at most $\left(n+n^{\prime}\right)\left(n+n^{\prime}-1\right) n n^{\prime} / 2$ comparisons to find the greatest in $\boldsymbol{R}$, because there are $\left(n+n^{\prime}\right)(n+$ $\left.n^{\prime}-1\right) / 2$ possible two-summand patterns when all $n$ sums in $\boldsymbol{Y}$ have become summands after differential transformations. After that, to identify each two-summand pattern, it needs $\left(s+n+n^{\prime}\right)\left(s+n+n^{\prime}-1\right) / 2-1$ comparisons, where $s$ is the number of identified patterns. After a pattern is identified, all rows with the pattern need to be updated. For each pattern, it needs to go through at most $n$ rows. Hence it requires at most $2 n n^{\prime}$ comparisons. If the pattern is forced, it needs to go through all identified patterns, which requires at most $n^{\prime} s$ comparisons for one row and $n n^{\prime} s$ comparison for $n$ rows. It requires at most $n n^{\prime}\left(n^{\prime}-1\right) / 2$ additions to update $\boldsymbol{R}$. Under direct computation, $M$ requires at most $n n^{\prime}$ additions. By identifying one pattern, the number of additions increases by one while saving at least one addition than direct computation. Based on this observation, we deduce that there are at most $n n^{\prime} / 2$ identified patterns. Thus the number of comparisons required in Step 25 is at most $\sum_{s=0}^{n n^{\prime} / 2-1}\left(\left(s+n+n^{\prime}\right)\left(s+n+n^{\prime}-\right.\right.$ 1) $\left./ 2-1+2 n n^{\prime}+n n^{\prime} s\right) \approx O\left(n^{3} n^{\prime 3}\right)$. The number of required additions is at most $\sum_{s=0}^{n n^{\prime} / 2-1}\left(n n^{\prime}\left(n^{\prime}-1\right) / 2\right) \approx O\left(n^{2} n^{\prime 3}\right)$. Assuming additions have the same complexity as comparisons, it is negligible. To identify one reversal pattern needs $n n^{\prime}\left(n^{\prime}-1\right)$, and its complexity is also negligible compared to those of other parts. Hence the complexity of our CSE algorithm is $O\left(n^{3} n^{\prime 3}+n^{4}\right)$, or $O\left(n^{6}\right)$ assuming $n=n^{\prime}$.

The time complexity above is for one run of Algorithm 2 Since Algorithm 2 is probabilistic, it is necessary to run it multiple times to obtain good results. However, a very large number of runs is not necessary even for large problems, since the variance between different runs is relatively small in comparison with the total number of required additions.

The storage complexity of Algorithm 2 includes five parts: $M, D, R, K$, and the list of identified two-summand patterns. For $\boldsymbol{M}$, it is at most $n n^{\prime}$. For $\boldsymbol{D}$, it is $n^{2}$ and can be reduced to $n(n-1)$ since $\boldsymbol{D}_{i i}$ is not necessary. Since there are at most $n n^{\prime} / 2$ identified patterns, the storage of $\boldsymbol{R}$ is at most $\left(n n^{\prime} / 2+\right.$ $\left.n+n^{\prime}\right)\left(n n^{\prime} / 2+n+n^{\prime}-1\right) / 2$ and it takes at most $n n^{\prime}$ to keep the list of identified patterns. The three-dimensional array $\boldsymbol{K}$ requires at most $n$ times of $\boldsymbol{M}$. Hence the total storage complexity is at most $O\left(n^{2} n^{\prime 2}\right)$, or $O\left(n^{4}\right)$ assuming $n=n^{\prime}$.

Note that the upper bound $n n^{\prime} / 2$ of the number of identified 
patterns for an $n \times n^{\prime}$ matrix is usually not tight. For example, for a $1023 \times 1023$ matrix, only less than 30,000 patterns are identified in our simulation.

\section{RELATIONS AMONG VARIOUS CFFTS}

Our CSE algorithm can be used to reduce the additive complexities of various CFFTs. In this section, we will investigate their properties and establish the relations among them. This study also simplifies the analysis of their multiplicative and additive complexities as well as performance comparison in Section V

Let us first study the properties of a block diagonal matrix $\boldsymbol{L}=\operatorname{diag}\left(\boldsymbol{L}_{0}, \boldsymbol{L}_{1}, \ldots, \boldsymbol{L}_{l-1}\right)$, where $\boldsymbol{L}_{i}$ 's are all circulant matrices. Clearly, $\boldsymbol{L}_{i}$ 's are all symmetric and hence $\boldsymbol{L}$ is also symmetric. We formally present a result mentioned in [7] and [30, pp. 273], which can be proved easily by inspection.

Lemma 1. Given $\boldsymbol{L}=\operatorname{diag}\left(\boldsymbol{L}_{0}, \boldsymbol{L}_{1}, \ldots, \boldsymbol{L}_{l-1}\right)$ that is a block diagonal matrix where $\boldsymbol{L}_{i}$ 's are all circulant, its inverse $\boldsymbol{L}^{-1}=$ $\operatorname{diag}\left(\boldsymbol{L}_{0}^{-1}, \boldsymbol{L}_{1}^{-1}, \ldots, \boldsymbol{L}_{l-1}^{-1}\right)$ is also a block diagonal matrix where $\boldsymbol{L}_{i}^{-1}$ 's are all circulant. Furthermore, suppose $\boldsymbol{L}_{i}$ is generated by $\gamma_{i}$ and $\boldsymbol{b}_{i}=\left(\gamma_{i}, \gamma_{i}^{2}, \ldots, \gamma_{i}^{2^{m_{i}-1}}\right)$ is a normal basis, then $\boldsymbol{L}_{i}^{-1}$ is a circulant matrix generated by $\beta_{i}$, where $\left(\beta_{i}, \beta_{i}^{2}, \ldots, \beta_{i}^{2^{m_{i}-1}}\right)$ is the dual basis of $\boldsymbol{b}_{i}$.

Thus, for DCFFTs and SCFFTs $\boldsymbol{L}_{i} \boldsymbol{f}_{i}$ is a cyclic convolution and can be calculated by the bilinear form $\boldsymbol{Q}_{i}\left(\boldsymbol{R}_{i} \boldsymbol{b}_{i} \cdot \boldsymbol{P}_{i} \boldsymbol{f}_{i}\right)=$ $\boldsymbol{Q}_{i}\left(\boldsymbol{c}_{i} \cdot \boldsymbol{P}_{i} \boldsymbol{f}_{i}\right)$ [12]-[15], where $\boldsymbol{b}_{i}=\left(\gamma_{i}, \gamma_{i}^{2}, \ldots, \gamma_{i}^{2^{m_{i}-1}}\right)$. For ICFFTs, by Lemma $1 \boldsymbol{L}_{i}^{-1} \boldsymbol{f}_{i}$ is also a cyclic convolution given by the bilinear form $\boldsymbol{Q}_{i}\left(\boldsymbol{R}_{i}\left(\beta_{i}, \beta_{i}^{2}, \ldots, \beta_{i}^{2^{m_{i}-1}}\right) \cdot \boldsymbol{P}_{i} \boldsymbol{f}_{i}\right)=$ $\boldsymbol{Q}_{i}\left(\boldsymbol{c}_{i}^{*} \cdot \boldsymbol{P}_{i} \boldsymbol{f}_{i}\right)$. There are different bilinear forms of cyclic convolution and all of them can be used in CFFTs. Henceforth, we assume that the same bilinear forms $\left(\boldsymbol{P}_{i}\right.$ 's and $\boldsymbol{Q}_{i}$ 's) are used in all CFFTs. In this paper, we focus on the CFFTs with the following forms:

$$
\begin{aligned}
\operatorname{DCFFT} \quad \boldsymbol{F} & =\boldsymbol{A} \boldsymbol{L} \boldsymbol{f}^{\prime} \\
& =\boldsymbol{A} \boldsymbol{Q}\left(\boldsymbol{c} \cdot \boldsymbol{P} \boldsymbol{f}^{\prime}\right) \\
\mathrm{SCFFT} \quad \boldsymbol{F}^{\prime} & =\boldsymbol{L}^{T} \boldsymbol{A}^{\prime T} \boldsymbol{f}^{\prime} \\
& =\boldsymbol{P}^{T}\left(\boldsymbol{c} \cdot\left(\boldsymbol{A}^{\prime} \boldsymbol{Q}\right)^{T} \boldsymbol{f}^{\prime}\right) \\
\mathrm{ICFFT} \quad \boldsymbol{F}^{\prime \prime} & =\boldsymbol{L}^{-1} \boldsymbol{A}^{-1} \boldsymbol{f} \\
& =\boldsymbol{P}^{T}\left(\boldsymbol{c}^{*} \cdot \boldsymbol{Q}^{T} \boldsymbol{A}^{-1} \boldsymbol{f}\right)
\end{aligned}
$$

where $\boldsymbol{Q}$ and $\boldsymbol{P}$ are binary matrices and usually sparse, and $\boldsymbol{A}$ is a dense binary square matrix. Note that the equality (3) is due to $\boldsymbol{L}=\boldsymbol{Q} \boldsymbol{C P}$ where $\boldsymbol{C}=\operatorname{diag}\left(c_{0}, c_{1}, \ldots, c_{n-1}\right)$; the equality (4) follows (3) and is a direct application of Lemma 1 Due to the symmetric properties of $\boldsymbol{L}$ and $\boldsymbol{L}^{-1}$, the above CFFTs have alternative forms: DCFFTs are also given by $\boldsymbol{F}=\boldsymbol{A} \boldsymbol{P}^{T}\left(\boldsymbol{c} \cdot \boldsymbol{Q}^{\boldsymbol{T}} \boldsymbol{f}^{\prime}\right)$; SCFFTs are also given by $\boldsymbol{F}^{\prime}=\boldsymbol{Q}\left(\boldsymbol{c} \cdot\left(\boldsymbol{A}^{\prime} \boldsymbol{Q}^{T}\right)^{T} \boldsymbol{f}^{\prime}\right)$; ICFFTs are also given by $\boldsymbol{F}^{\prime \prime}=$ $\boldsymbol{Q}\left(\boldsymbol{c}^{*} \cdot \boldsymbol{P} \boldsymbol{A}^{-1} \boldsymbol{f}\right)$. However, these alternative forms can be considered as the forms in (2), (3), and (4) with different $\boldsymbol{P}$ and $Q$ matrices. Since we assume all the bilinear forms are the same, we will not consider the alternative forms further.

We observe that all CFFTs in (2), (3), and (4) are determined by two factors. First, they all depend on the order of cyclotomic cosets, i.e., the coset leaders $k_{i}$ 's, which in turn determine the coset size $m_{i}$ 's. As in [6], we assume the same normal basis is used for all cyclotomic cosets of the same size. Hence, all CFFTs also depend on the normal basis selected for each subfield $\mathrm{GF}\left(2^{m_{i}}\right)$. For simplicity, we denote the collections of DCFFTs, SCFFTs, and ICFFTs for different $k_{i}$ 's, $m_{i}$ 's and the normal bases as $\mathcal{D}, \mathcal{S}$, and $\mathcal{I}$, respectively. Next, we investigate the impact on computational complexities of CFFTs by the two factors above. We will consider first multiplicative complexities and then additive complexities.

Lemma 2. Assuming that the same bilinear forms are used, DCFFTs, SCFFTs, and ICFFTs as defined in (2), (3), and (4) have the same multiplicative complexities.

Proof: The multiplicative complexity is determined by the number of non-one entries in $c$ in DCFFTs and SCFFTs or $c^{*}$ in ICFFTs (all elements in $c$ or $c^{*}$ are non-zero). Since using normal bases, the number of 1 's in $c$ and $c^{*}$ are both the number of all-one rows in all $\boldsymbol{R}_{i}$ 's. Thus the multiplicative complexity is independent of the choices of normal bases and independent of the constant vectors $c$ or $c^{*}$.

The additive complexities of all CFFTs are due to the matrixvector multiplications needed in CFFTs. Clearly, the number of additions required to compute any matrix-vector multiplication $\boldsymbol{Y}=\boldsymbol{M} \boldsymbol{X}$ varies with the implementation. In the following, we will consider additive complexities under direct computation. As pointed out in Section III-C to compute $\boldsymbol{Y}=$ $\boldsymbol{M} \boldsymbol{X}$ by direct computation, it needs $W(\boldsymbol{M})-n$ additions. In some cases the additive complexities of two matrix-vector multiplications can be related regardless of implementation. We say two matrix-vector multiplications are additively equivalent if one matrix-vector multiplication can achieve any additive complexity the other can, and vice versa. An important case of additive equivalence is given in the following lemma without proof.

Lemma 3. If two binary matrices $M$ and $\boldsymbol{M}^{\prime}$ satisfy $\boldsymbol{M}^{\prime}=$ $\Pi M \Pi^{\prime}$, where $\Pi$ and $\Pi^{\prime}$ are two permutation matrices, then the matrix-vector multiplications defined by $M$ and $M^{\prime}$ are additively equivalent.

With a slight abuse of terminology, we say two CFFTs are additively equivalent when their corresponding matrices are additively equivalent. By a straightforward proof, we have the following property:

Lemma 4. For any two CFFTs in $\mathcal{D}$ that differ only in $k_{i}$ 's and $m_{i}$ 's, their $\boldsymbol{A}$ 's and $\boldsymbol{L}$ 's are additively equivalent, respectively. Thus, the two CFFTs in $\mathcal{D}$ are additively equivalent. The same property holds for $\mathcal{S}$ and $\mathcal{I}$.

We now consider additive complexities for all CFFTs when normal bases vary, too.

Lemma 5. All CFFTs in D have the same additive complexity under direct computation. So do those in $\mathcal{S}$ and $\mathcal{I}$, respectively.

Proof: It suffices to prove the first part, and the arguments for $\mathcal{S}$ and $\mathcal{I}$ are similar. First, since different orders of cosets result in additively equivalent DCFFTs due to Lemma 4, we assume the same order of cosets and consider only different normal bases without loss of generality. Realizing that differ- 
ent normal bases would not change $P$ and $Q$ in (2), we focus on how different normal bases impact $\boldsymbol{A} \boldsymbol{Q}$. Expressing $\boldsymbol{A}$ as $\left[\boldsymbol{A}_{0}\left|\boldsymbol{A}_{1}\right| \cdots \mid \boldsymbol{A}_{l-1}\right]$ where $\boldsymbol{A}_{i}$ is a $\left(2^{m}-1\right) \times m_{i}$ binary matrix, $\boldsymbol{F}=\boldsymbol{A} \boldsymbol{L} \boldsymbol{f}^{\prime}=\left[\boldsymbol{A}_{0} \boldsymbol{L}_{0}\left|\boldsymbol{A}_{1} \boldsymbol{L}_{1}\right| \cdots \mid \boldsymbol{A}_{l-1} \boldsymbol{L}_{l-1}\right] \boldsymbol{f}^{\prime}$. For each $\boldsymbol{A}_{i}$, the rows are $\left(2^{m}-1\right) /\left(2^{m_{i}}-1\right)$ copies of the set of $m_{i}$-bit row vectors with all combinations except all zeros. Thus $\boldsymbol{A}_{i}$ 's corresponding to different normal bases in $\operatorname{GF}\left(2^{m_{i}}\right)$ are equivalent up to permutation. Recall that $\boldsymbol{Q}$ is a block matrices for which the blocks off the diagonal are zero matrices and the diagonal blocks are $Q_{i}$ 's. Thus, $A Q=\left[A_{0} Q_{0}\left|A_{1} Q_{1}\right| \cdots \mid A_{l-1} Q_{l-1}\right]$. Thus $A Q$ 's corresponding to different normal bases also have the same additive complexity under direct computation. Hence all DCFFTs in $\mathcal{D}$ have the same additive complexity under direct computation.

From Lemma 1, we establish a relation between $\mathcal{I}$ and $\mathcal{S}$.

Lemma 6. Given an ICFFT $\boldsymbol{F}^{\prime \prime}=\boldsymbol{L}^{-1} \boldsymbol{A}^{-1} \boldsymbol{f}$, there exists an SCFFT $\boldsymbol{F}^{\prime}=\boldsymbol{L}^{\prime} \boldsymbol{A}^{\prime T} \boldsymbol{f}^{\prime}$ such that $\boldsymbol{L}^{\prime}=\boldsymbol{L}^{-1}$ and $\boldsymbol{A}^{\prime T}$ and $\boldsymbol{A}^{-1}$ are equivalent up to permutation, and vice versa.

Proof: It suffices to show the first part, and the argument for the second part is similar. For a DCFFT given by $\boldsymbol{F}=\boldsymbol{A} \boldsymbol{L} \boldsymbol{\Pi} \boldsymbol{f}$, the transform $\boldsymbol{F}^{*}=\boldsymbol{\Pi}^{-1} \boldsymbol{L}^{-1} \boldsymbol{A}^{-1} \boldsymbol{f}$ is another DFT, where $\boldsymbol{F}^{*}=\left(F_{0}, F_{n-1}, F_{n-2}, \ldots, F_{1}\right)=\boldsymbol{\Pi}^{*} \boldsymbol{F}$ and $\boldsymbol{\Pi}^{*}$ is a permutation matrix. Given an ICFFT $\boldsymbol{F}^{\prime \prime}=$ $\boldsymbol{L}^{-1} \boldsymbol{A}^{-1} \boldsymbol{f}$, clearly $\boldsymbol{F}^{\prime \prime}=\boldsymbol{\Pi} \boldsymbol{F}^{*}=\boldsymbol{\Pi} \boldsymbol{\Pi}^{*} \boldsymbol{F}$. Suppose the indices of the components of $\boldsymbol{F}^{\prime}=\boldsymbol{\Pi} \boldsymbol{F}$ are in the order as $\left(k_{0}, k_{0} 2, \ldots, k_{0} 2^{m_{0}-1}, \ldots, k_{l-1} 2^{m_{l-1}-1}\right) \bmod n$, then the indices of the components of $\boldsymbol{F}^{\prime \prime}=\boldsymbol{\Pi} \boldsymbol{F}^{*}$ are in the order as $(n-$ $\left.k_{0}, n-k_{0} 2, \ldots, n-k_{0} 2^{m_{0}-1}, \ldots, n-k_{l-1} 2^{m_{l-1}-1}\right) \bmod n$. Note that both modulo operations above are componentwise. Since $n-k_{i} 2^{j} \equiv\left(n-k_{i}\right) 2^{j} \bmod n, \boldsymbol{F}^{\prime \prime}$ is also ordered in cyclotomic cosets. Let us consider an SCFFT with the same order of cyclotomic cosets: $\boldsymbol{F}^{\prime \prime}=\boldsymbol{L}^{\prime \prime} \boldsymbol{A}^{\prime \prime T} \boldsymbol{f}^{\prime \prime}$ where $\boldsymbol{f}^{\prime \prime}=\boldsymbol{\Pi} \boldsymbol{\Pi}^{*} \boldsymbol{f}$. Note that the order of the cyclotomic cosets sizes $m_{i}$ remains the same in $\boldsymbol{L}^{\prime \prime}$ and $\boldsymbol{L}^{-1}$. Thus by Lemma 1 there exist normal bases such that $\boldsymbol{L}^{\prime \prime}=\boldsymbol{L}^{-1}$. Choosing such normal bases, we construct an SCFFT $\boldsymbol{F}^{\prime \prime}=\boldsymbol{L}^{-1} \boldsymbol{A}^{\prime \prime T} \boldsymbol{f}^{\prime \prime}=\boldsymbol{L}^{-1} \boldsymbol{A}^{-1} \boldsymbol{f}$. Thus $\boldsymbol{L}^{-1}\left(\boldsymbol{A}^{\prime \prime T} \boldsymbol{\Pi} \boldsymbol{\Pi}^{*}-\boldsymbol{A}^{-1}\right) \boldsymbol{f}=0$ for arbitrary $\boldsymbol{f}$ and full rank $\boldsymbol{L}^{-1}$. Hence $\boldsymbol{A}^{-1}=\boldsymbol{A}^{\prime \prime T} \boldsymbol{\Pi}^{*} \boldsymbol{\Pi}$.

Note that Lemma 6 holds regardless of implementation. Since this mapping exists for any ICFFTs or SCFFTs, Lemma6 implies that ICFFTs and SCFFTs are additively equivalent.

Finally, we are ready to relate the additive complexities of all CFFTs under direct computation.

Lemma 7. The DCFFTs, SCFFTs, and ICFFTs in (2), (3), and (4) all have the same additive complexity under direct computation.

Proof: Due to Lemma 5, it is sufficient to show that the additive complexities of two CFFTs of different types are the same, which holds for an SCFFT and an ICFFT by Lemmas 5 and 6 . Now let us show it is the same for a DCFFT and an SCFFT.

In length- $n$ DCFFTs, $\boldsymbol{A}$ is an $n \times n$ matrix, $\boldsymbol{Q}$ is an $n \times n^{\prime}$ matrix $\left(n^{\prime}>n\right)$, and $\boldsymbol{P}$ is an $n^{\prime} \times n$ matrix. Under direct computation, the number of required additions for a DCFFT defined in (2) is $W(\boldsymbol{A} \boldsymbol{Q})-n+W(\boldsymbol{P})-n^{\prime}$. Since $\boldsymbol{f}^{\prime}=\boldsymbol{\Pi} \boldsymbol{f}$, we have $\boldsymbol{F}^{\prime}=\boldsymbol{A}^{\prime} \boldsymbol{Q}\left(\boldsymbol{c} \cdot \boldsymbol{P} \boldsymbol{f}^{\prime}\right)$, where $\boldsymbol{F}^{\prime}=\boldsymbol{\Pi} \boldsymbol{F}$ and $\boldsymbol{A}^{\prime}=$ $\boldsymbol{\Pi} \boldsymbol{A}$. For an SCFFT $\boldsymbol{F}^{\prime}=\boldsymbol{P}^{T}\left(\boldsymbol{c} \cdot\left(\boldsymbol{A}^{\prime} \boldsymbol{Q}\right)^{T} \boldsymbol{f}^{\prime}\right)$, the additive complexity under direct computation is $W\left(\left(\boldsymbol{A}^{\prime} \boldsymbol{Q}\right)^{T}\right)-n^{\prime}+$ $W\left(\boldsymbol{P}^{T}\right)-n$. Since $\boldsymbol{A}^{\prime} \boldsymbol{Q}=\boldsymbol{\Pi} \boldsymbol{A} \boldsymbol{Q}$, so $\boldsymbol{A}^{\prime} \boldsymbol{Q}$ and $\boldsymbol{A} \boldsymbol{Q}$ have the same number of 1 's. Since matrix transpose does not change the number of 1's, $W\left(\left(\boldsymbol{A}^{\prime} \boldsymbol{Q}\right)^{T}\right)=W(\boldsymbol{A} \boldsymbol{Q})$ and $W\left(\boldsymbol{P}^{T}\right)=$ $W(\boldsymbol{P})$. Hence any DCFFTs in (2) and any SCFFTs in (3) have the same additive complexity under direct computation. An alternative direct computation for both DCFFTs and SCFFTs is to multiply $\boldsymbol{A}$ and $\boldsymbol{Q}$ separately. It is easy to verify that the conclusion is the same.

\section{CFFTs with Reduced Additive Complexities}

Using Algorithm 2, we construct CFFTs with reduced additive complexities for lengths $2^{m}-1$ up to 1023 , and we present their complexities in Table【. CFFTs of length beyond 1023 are not considered because for two reasons: first, lengths beyond 1023 are rarely needed for the primary application considered in this paper, Reed-Solomon decoders; second, efficient cyclic convolutions for CFFTs of longer lengths (for example, 11point cyclic convolution for length-2047 CFFTs) are not available in [14]-[16]. For all our CFFTs, the cyclotomic cosets are ordered by their leaders; for cyclic convolutions of lengths up to nine, we use the bilinear forms provided in [16], and we construct a length-10 cyclic convolution based on those of lengths two and five, by the Agarwal-Cooley algorithm [31]; the primitive polynomials and vector-space representations in [32, Sec. B.3] are used for all fields; for each field, we choose the normal basis whose leader is the smallest power of the primitive element. We observe that the multiplicative complexities are the same for all CFFTs due to Lemma 2. Due to Lemma 6. SCFFTs and ICFFTs are additively equivalent, and the additive complexities of both SCFFTs and ICFFTs are presented together in Table I We also observe that SCFFTs and ICFFTs require more additions than DCFFTs, and the reason for this was given in [8].

TABLE I

COMPLEXITIES OF FuLL CyCLOTOMIC FFTS

\begin{tabular}{|c|c|c|c|c|c|c|}
\hline \multirow{2}{*}{$n$} & \multirow{2}{*}{ Mult. } & \multicolumn{5}{|c|}{ Additions } \\
\cline { 3 - 7 } & & \multicolumn{2}{|c|}{ DCFFT } & \multicolumn{2}{|c|}{ SCFFT/ICFFT } \\
\cline { 3 - 7 } & & Ours & {$[6]$} & Ours & {$[7]$} & {$[8]$} \\
\hline 7 & 6 & 24 & 25 & 24 & 24 & - \\
\hline 15 & 16 & 74 & 77 & 76 & - & 91 \\
\hline 31 & 54 & 299 & 315 & 307 & - & - \\
\hline 63 & 97 & 759 & 805 & 804 & - & - \\
\hline 127 & 216 & 2576 & 2780 & 3117 & - & - \\
\hline 255 & 586 & 6736 & 7919 & 6984 & - & - \\
\hline 511 & 1014 & 23130 & 26643 & 27192 & - & \\
\hline 1023 & 2827 & 75360 & - & 77276 & - & - \\
\hline
\end{tabular}

In Table \, we also compare the additive complexities of our CFFTs to those in [6]-[8], the best results of CFFTs in the open literature to our knowledge 2 . In Table \ some entries are blank due to unavailability of comparable data: the additive complexity of DCFFT of length 1023 is not provided in [6]; only length-7 ICFFT was provided in [7] and only length15 SCFFTs was provided in [8]. For length-7 FFT, both our

\footnotetext{
${ }^{2}$ A length-15 DCFFT with 76 additions was reported in [16].
} 
TABLE II

Complexities OF Full FFTS

\begin{tabular}{|c|c|c|c|c|c|c|c|c|c|c|c|c|c|c|c|c|c|c|}
\hline \multirow{2}{*}{$n$} & \multicolumn{2}{|c|}{ Horner's rule } & \multicolumn{2}{|c|}{ Goertzel's alg. } & \multicolumn{2}{|c|}{ [5] } & \multicolumn{3}{|c|}{ [33] } & \multicolumn{3}{|c|}{ Bergland's alg. } & \multicolumn{3}{|c|}{ Prime-factor [2] } & \multicolumn{3}{|c|}{ Our DCFFTs } \\
\hline & Mult. & Add. & Mult. & Add. & Mult. & Add. & Mult. & Add. & Total & Mult. & Add. & Total & Mult. & Add. & Total & Mult. & Add. & Total \\
\hline 7 & 36 & 42 & 12 & 42 & 6 & 26 & 29 & 29 & 174 & - & - & - & 9 & 37 & 82 & 6 & 24 & 54 \\
\hline 15 & 196 & 210 & 38 & 210 & 16 & 100 & 41 & 97 & 384 & - & - & - & - & - & - & 16 & 74 & 186 \\
\hline 31 & 900 & 930 & 120 & 930 & 60 & 388 & 289 & 289 & 2890 & - & - & - & 108 & 612 & 1584 & 54 & 299 & 785 \\
\hline 63 & 3844 & 3906 & 282 & 3906 & 97 & 952 & 801 & 801 & 9612 & - & - & - & - & - & - & 97 & 759 & 1826 \\
\hline 127 & 15876 & 16002 & 756 & 16002 & 468 & 3737 & 2113 & 2113 & 29582 & - & - & - & - & - & - & 216 & 2576 & 5384 \\
\hline 255 & 64516 & 64770 & 1718 & 64770 & 646 & 35503 & 1665 & 5377 & 30352 & 5610 & 5610 & 89760 & 1135 & 3887 & 20902 & 586 & 6736 & 15526 \\
\hline 511 & 260100 & 260610 & 4044 & 260610 & - & - & 13313 & 13313 & 239634 & 39858 & 39858 & 717444 & 6516 & 17506 & \begin{tabular}{|l|}
128278 \\
\end{tabular} & 1014 & 23130 & 40368 \\
\hline 1023 & 1044484 & 1045506 & 9032 & 1045506 & - & - & 32257 & 32257 & 645140 & 42966 & 42966 & 859320 & 5915 & 30547 & 142932 & 2827 & 75360 & 129073 \\
\hline
\end{tabular}

DCFFT and SCFFT achieve the smallest additive complexity of the ICFFT in [7]; for lengths 15, 31, 63, and 127, our CFFTs have additive complexities $4 \%, 5 \%, 6 \%$, and $7 \%$ smaller than those reported in [6]; for lengths 255 and 511, our CFFTs reduce additive complexities by $15 \%$ and $13 \%$, respectively, than their counterparts in [6]. To compare our length-7 DCFFT with that in [6], see Appendix A

We also compare our results to other FFT algorithms in Table [I] For Horner's rule [34], Goertzel's algorithm [14], Zakharova's method [5], the complexities are reproduced from [6] except that the complexities of length-1023 FFTs are reproduced from [2]; the complexities of Bergland's algorithm [35] and the prime-factor FFTs [2] are obtained from [2], [3]. For reference, we also consider the algorithm proposed by Wang and Zhu [33], which is known to be asymptotically fast, and its complexities are obtained from [33, eq. (11) and (12)].

Since all the algorithms require both multiplicative and additive complexities, it is clear that a metric for the total complexities is needed for comparison. We use a weighted sum of the additive and multiplicative complexities as the metric, assuming the complexity of each multiplication is $2 m-1$ times as that of an addition. Our assumption is based on both hardware and software considerations. In hardware implementation, a multiplier over $\operatorname{GF}\left(2^{m}\right)$ generated by trinomials requires $m^{2}-1$ XOR and $m^{2}$ AND gates (see, e.g., [36]), while an adder requires $m$ XOR gates. Assuming that XOR and AND gates require the same area, the area complexity of a field multiplier is $2 m$ times that of an adder over $\operatorname{GF}\left(2^{m}\right)$. In software implementation, the complexity can be measured by the number of word-level operations (see, for example, [37]). Using the shift and add method as in [37], a multiplication requires $m-1$ shift and $m$ XOR word-level operations, respectively while an addition needs only one XOR wordlevel operation. Whenever the complexity of a multiplication is more than $2 m-1$ times as complex as that of an addition (for example, in the hardware implementation described above), our assumption above underestimates the relative complexity of multiplications and hence puts our results in a disadvantage in comparison to other FFT algorithms since CFFTs have reduced multiplicative complexities. We would also like to point out the similarity between our metric and the one used in [33], where the multiplication over $\operatorname{GF}\left(2^{m}\right)$ was treated $2 m$ times as complex as an addition.

The total complexities of Horner's rule, Goertzel's algorithm, and [5] are not presented in Table $\Pi$ since the advantage in complexities of our CFFTs over Horner's rule, Goertzel's algorithm, and [5] is clear: our CFFTs require fewer multiplications and fewer additions; the savings achieved by our CFFTs are very significant, and in some cases the multiplicative complexities of our CFFTs are only small fractions of other algorithms. We remark that the multiplicative complexities of Zakharova's method are closer to those of CFFTs, which is not surprising given their similarities [6]. The total complexities of [33], Bergland's algorithm, the prime-factor FFTs [2] and our CFFTs are presented in Table III since in comparison to these algorithms our CFFTs have smaller multiplicative complexities but higher additive complexities. In comparison to [33], our CFFTs achieve total complexity savings of $69 \%, 52 \%, 73 \%, 81 \%, 82 \%, 49 \%, 83 \%$ and $80 \%$ for lengths $7,15, \ldots, 1023$, respectively. For lengths 255,511 , and 1023 , our CFFTs achieve total complexity savings of $83 \%$, $94 \%$, and $85 \%$ over Bergland's algorithm, and $26 \%, 69 \%$, and $10 \%$ over the prime-factor FFTs [2], respectively.

We remark that, as in many previous works (see, for example, [6]-[8]), only the multiplications and additions are considered in the complexity comparison. This is reasonable if the CFFTs are implemented by combinational logic, and the required numbers of multiplications and additions translate to the numbers of finite field multipliers and adders in combinational logic. Under the same assumption, memory overhead and intermediate memory access are not considered in the comparison above. This would not be the case if CFFTs were implemented in software, but this is beyond the scope of this paper.

\section{ACKNOWLEDGMENT}

The authors would like to thank Prof. P. Trifonov for providing details of CFFTs. They are grateful to Prof. M.D. Wagh for introducing them to his fast convolution algorithms. They would also thank Prof. P.D. Chen for valuable discussions. The authors would also like to thank the reviewers for their constructive comments, which have resulted in significant improvements in the manuscript.

\section{APPENDIX A LENGTH-7 DCFFT}

- Pre-additions $\boldsymbol{p}=\left(p_{0}, p_{1}, \ldots, p_{8}\right)^{T}=\boldsymbol{P} \boldsymbol{f}^{\prime}$ require 8 additions: $p_{0}=f_{0}, p_{2}=f_{2}+f_{4}, p_{3}=f_{1}+f_{2}, p_{4}=$ $f_{1}+f_{4}, p_{1}=p_{2}+f_{1}, p_{6}=f_{6}+f_{5}, p_{7}=f_{3}+f_{6}$, $p_{8}=f_{3}+f_{5}$, and $p_{5}=p_{6}+f_{3}$. 
- Pointwise multiplications $\boldsymbol{g}=\left(g_{0}, g_{1}, \ldots, g_{8}\right)^{T}=\boldsymbol{c} \cdot \boldsymbol{p}$, where $c=\left(1,1, \alpha, \alpha^{2}, \alpha^{4}, 1, \alpha, \alpha^{2}, \alpha^{4}\right)^{T}$, need 6 multiplications

- Post-additions $\boldsymbol{F}=\left(F_{0}, F_{1}, \ldots, F_{6}\right)^{T}=\boldsymbol{A} \boldsymbol{Q g}$ require 16 additions: $t_{0}=g_{3}+g_{4}, t_{1}=g_{0}+g_{1}, t_{2}=g_{1}+g_{5}$, $F_{0}=g_{0}+t_{2}, t_{3}=g_{2}+g_{4}, t_{4}=g_{8}+t_{3}, t_{5}=g_{7}+t_{4}$, $F_{5}=t_{1}+t_{5}, t_{6}=g_{6}+t_{4}, t_{7}=t_{1}+t_{6}, F_{6}=t_{0}+t_{7}$, $F_{3}=F_{6}+t_{5}, t_{8}=t_{3}+t_{2}, F_{2}=F_{3}+t_{8}, F_{1}=F_{2}+t_{6}$, and $F_{4}=t_{2}+t_{7}$.

\section{REFERENCES}

[1] R. E. Blahut, "Transform techniques for error control codes," IBM J. Res. Develop., vol. 23, no. 3, pp. 299-315, May 1979.

[2] T. K. Truong, P. D. Chen, L. J. Wang, I. S. Reed, and Y. Chang, "Fast, prime factor, discrete Fourier transform algorithms over $\mathrm{GF}\left(2^{m}\right)$ for $8 \leq m \leq 10$," Inf. Sci., vol. 176, no. 1, pp. 1-26, Jan. 2006.

[3] T. K. Truong, P. D. Chen, L. J. Wang, and T. C. Cheng, "Fast transform for decoding both errors and erasures of Reed-Solomon codes over GF $\left(2^{m}\right)$ for $8 \leq m \leq 10$," IEEE Trans. Commun., vol. 54, no. 2, pp. 181-186, Feb. 2006.

[4] T.-C. Lin, T. K. Truong, and P. D. Chen, "A fast algorithm for the syndrome calculation in algebraic decoding of Reed-Solomon codes," IEEE Trans. Commun., vol. 55, no. 12, pp. 1-5, Dec. 2007.

[5] T. G. Zakharova, "Fourier transform evaluation in fields of characteristic 2," Probl. Inf. Transm., vol. 28, no. 2, pp. 154-167, 1992.

[6] P. V. Trifonov and S. V. Fedorenko, "A method for fast computation fo the Fourier transform over a finite field," Probl. Inf. Transm., vol. 39, no. 3, pp. 231-238, 2003. [Online]. Available: http://dcn.infos.ru/ petert/papers/fftEng.pdf

[7] E. Costa, S. V. Fedorenko, and P. V. Trifonov, "On computing the syndrome polynomial in Reed-Solomon decoder," Euro. Trans. Telecomms., vol. 15, no. 4, pp. 337-342, 2004. [Online]. Available: http://den.infos.ru/ petert/papers/syndromes_ett.pdf

[8] S. V. Fedorenko, "A method of computation of the discrete Fourier transform over a finite field," Probl. Inf. Transm., vol. 42, no. 2, pp. 139-151, 2006.

[9] M. R. Garey and D. S. Johnson, Computers and Intractability: A Guide to the Theory of NP-Completeness, ser. Books in the Mathematical Sciences. San Francisco, CA: W. H. Freeman, 1979.

[10] P. Cappello and K. Steiglitz, "Some complexity issues in digital signal processing," IEEE Trans. Acoust., Speech, Signal Process., vol. 32, no. 5, pp. 1037-1041, Oct. 1984.

[11] R. A. Horn and C. R. Johnson, Matrix Analysis. New York, NY: Cambridge Univ. Press, 1985.

[12] S. Winograd, "Some bilinear forms whose multiplicative complexity depends on the field of constants," Math. Syst. Theory, vol. 10, no. 1, pp. 169-180, 1977.

[13] M. D. Wagh and S. D. Morgera, "A new structured design method for convolutions over finite fields, Part I," IEEE Trans. Inf. Theory, vol. 29, no. 4, pp. 583-595, Jul. 1983.

[14] R. E. Blahut, Theory and Practice of Error Control Codes. Reading, MA: Addison-Wesley, 1983.

[15] — Fast Algorithms for Digital Signal Processing. Reading, MA: Addison-Wesley, 1984.

[16] P. Trifonov, private communication.

[17] — - "Matrix-vector multiplication via erasure decoding," in Proc. XI Int. Symp. Problems of Redundancy in Information and Control Systems, Saint-Petersburg, Russia, Jul. 2007, pp. 104-108. [Online]. Available: http://dcn.infos.ru/ petert/papers/mo.pdf

[18] A. V. Aho, J. E. Hopcroft, and J. D. Ullman, The Design and Analysis of Computer Algorithms. Reading, MA: Addison-Wesley, 1974.

[19] M. Potkonjak, M. B. Srivastava, and A. P. Chandrakasan, "Multiple constant multiplications: efficient and versatile framework and algorithms for exploring common subexpression elimination," IEEE Trans. Comput.-Aided Design Integr. Circuits Syst., vol. 15, no. 2, pp. 151-165, Feb. 1996.

[20] R. Paško, P. Schaumont, V. Derudder, S. Vernalde, and D. Ďuračková, "A new algorithm for elimination of common subexpressions," IEEE Trans. Comput.-Aided Design Integr. Circuits Syst., vol. 18, no. 1, pp. 58-68, Jan. 1999.

[21] R. I. Hartley, "Subexpression sharing in filters using canonic signed digit multipliers," IEEE Trans. Circuits Syst. II, vol. 43, no. 10, pp. 677-688, Oct. 1996.
[22] M. Martínez-Peiró, E. I. Boemo, and L. Wanhammar, "Design of high-speed multiplierless filters using a nonrecursive signed common subexpression algorithm," IEEE Trans. Circuits Syst. II, vol. 49, no. 3, pp. 196-203, Mar. 2002.

[23] R. Mahesh and A. P. Vinod, "A new common subexpression elimination algorithm for realizing low-complexity higher order digital filters," IEEE Trans. Comput.-Aided Design Integr. Circuits Syst., vol. 27, no. 2, pp. 217-229, Feb. 2008.

[24] A. G. Dempster and M. D. Macleod, "Use of minimum-adder multiplier blocks in FIR digital filters," IEEE Trans. Circuits Syst. II, vol. 42, no. 9, pp. 569-577, Sep. 1995.

[25] C.-H. Chang, J. Chen, and A. P. Vinod, "Information theoretic approach to complexity reduction of FIR filter design," IEEE Trans. Circuits Syst. $I$, to be published.

[26] X. Zhang and K. K. Parhi, "Implementation approaches for the Advanced Encryption Standard algorithm," IEEE Circuits Syst. Mag., vol. 2, no. 4, pp. 24-46, 2002.

[27] M. D. Macleod and A. G. Dempster, "Common subexpression elimination algorithm for low-cost multiplierless implementation of matrix multipliers," Electron. Lett., vol. 40, no. 11, pp. 651-652, May 2004.

[28] O. Gustafsson and M. Olofsson, "Complexity reduction of constant matrix computations over the binary field," in Proc. Int. Workshop Arithmetic Finite Fields (WAIFI'07), ser. Lecture Notes in Computer Science, vol. 4547, Madrid, Spain, Jun. 2007, pp. 103-115.

[29] D. P. Bertsekas, Dynamic Programming and Optimal Control. Belmont, MA: Athena Scientific, 1995.

[30] J. Hong and M. Vetterli, "Computing $m$ DFT's over GF $(q)$ with one DFT over $\operatorname{GF}\left(q^{m}\right)$," IEEE Trans. Inf. Theory, vol. 49, no. 1, pp. 271274, Jan. 1993.

[31] R. Agarwal and J. Cooley, "New algorithms for digital convolution," IEEE Trans. Acoust., Speech, Signal Process., vol. 25, no. 5, pp. 392410, Oct. 1977.

[32] S. B. Wicker, Error Control Systems for Digital Communications and Storage. Upper Saddle River, NJ: Prentice Hall, 1995.

[33] Y. Wang and X. Zhu, "A fast algorithm for the Fourier transform over finite fields and its VLSI implementation," IEEE J. Sel. Areas Commun., vol. 6, no. 3, pp. 572-577, Apr. 1988.

[34] S. B. Wicker and V. K. Bhargava, Eds., Reed-Solomon Codes and Their Applications. New York, NY: IEEE Press, 1994.

[35] E. O. Brigham, The Fast Fourier Transform and Its Applications. Englewood, NJ: Prentice-Hall, 1988.

[36] B. Sunar and Ç. K. Koç, "Mastrovito multiplier for all trinomials," IEEE Trans. Comput., vol. 48, no. 5, pp. 522-527, May 1999.

[37] A. Mahboob and N. Ikram, "Lookup table based multiplication technique for $\mathrm{GF}\left(2^{m}\right)$ with cryptographic significance," IEE Proc.-Commun., vol. 152, no. 6, pp. 965-974, Dec. 2005. 